\title{
Antioxidants and respiratory disease: the uric acid paradox
}

\author{
Seif 0 Shaheen
}

For humble individuals like myself, there is one poor comfort, which is this, viz. that gout, unlike any other disease, kills more rich men than poor, more wise men than simple. Thomas Sydenham.

In clinical practice, high blood concentrations of uric acid (UA) are generally thought to be a bad thing-primarily because of the risk of gout, but also because individuals with high UA concentrations are at increased risk of cardiovascular disease, metabolic syndrome and hypertension $^{1}$; these associations may reflect the fact that, under certain circumstances, UA can be pro-oxidant. ${ }^{2}$ Perhaps less appreciated are the potentially beneficial antioxidant properties of UA. ${ }^{3} \mathrm{UA}$ is present in the epithelial lining fluid (ELF) of the airways and contributes, along with other endogenous antioxidants, such as reduced glutathione, and dietary antioxidants, such as ascorbic acid, to antioxidant defences. ${ }^{4}$ Oxidative stress is thought to play an important role in the pathogenesis of asthma and $\mathrm{COPD}^{5}$ and may also be involved in the pathogenesis of lung cancer by causing DNA damage. ${ }^{6}$ This raises the interesting possibility that high concentrations of UA in the ELF may, by boosting antioxidant defences, reduce oxidative stress in the airways, thereby decreasing the risk of developing obstructive pulmonary disease and lung cancer; such beneficial effects of UA (and other antioxidants in the ELF) would be expected to be greatest in individuals with a high oxidant burden in the airways, such as smokers and those exposed to high levels of pollution.

To date, epidemiological research into the possible aetiological role of antioxidants in obstructive lung disease and lung cancer has focused particularly on the antioxidant vitamins, in the hope that preventive dietary interventions might be devised. However, this hope has not been realised. For example, despite evidence implicating a low intake of vitamin $\mathrm{C}$ in asthma, ${ }^{7}$ adults with asthma derived no clinical benefit from vitamin C

Correspondence to Professor Seif O Shaheen, Centre for Primary Care and Public Health, Blizard Institute, Barts and The London School of Medicine and Dentistry, 58 Turner Street, London E1 2AB, UK; s.shaheen@qmul.ac.uk supplementation compared with placebo. ${ }^{8}$ Similarly, although observational data have suggested that a diet rich in carotenoids may prevent the development of lung cancer, ${ }^{9}$ trial data have indicated that $\beta$ carotene supplementation increases the risk of lung cancer in smokers. ${ }^{10}$ There are a number of possible explanations for the discordance between the observational evidence and findings from randomised clinical trials. First, the observational findings may have arisen as a result of bias or confounding. The latter is particularly a problem in nutritional epidemiology; dietary habits are strongly socially patterned (with higher antioxidant intakes in higher socioeconomic groups) and nutrient intakes are highly correlated, thus the chances of spurious non-causal associations arising through residual or uncontrolled confounding are high. ${ }^{11}$ Second, the pulmonary oxidant/antioxidant paradigm may be over-simplistic (some antioxidants also have pro-oxidant properties) or fundamentally flawed. For example, the dogma that oxidants always contribute to inflammatory lung injury has been challenged, ${ }^{12}$ and in animals, antioxidants appear to accelerate progression of lung cancer. ${ }^{13}$ These observations can be viewed in the broader context of findings that challenge the free radical theory of ageing; a meta-analysis of randomised trials showed that antioxidant supplement use is associated with increased adult allcause mortality, ${ }^{14}$ and in an animal model, oxidative stress increased longevity. ${ }^{15}$ Third, it may be that the observational findings are correct, but for antioxidant nutrients to have beneficial effects they must be consumed in combination in food, thus enabling interactions, and not as single nutrients in pill form. In keeping with this idea, there is some epidemiological evidence that a 'prudent' diet, rich in antioxidants, is associated with higher lung function and a lower prevalence of COPD, especially in male smokers, ${ }^{16}$ and limited trial data suggest that short-term improvement in adult asthma might be achieved through a foodbased intervention to modify dietary antioxidant intake. ${ }^{17}$

Given that few epidemiological studies have investigated the role of endogenous antioxidants such as UA in the aetiology of respiratory disease, the paper by Horsfall and colleagues is to be welcomed. Exploiting a large primary care database, they retrospectively identified a cohort of individuals who had had serum UA measured, and set out to investigate the relation between serum UA and incident COPD and lung cancer, and whether associations were modified by smoking. ${ }^{18}$ After controlling for potential confounders, they found weak negative associations between serum UA and incident COPD and lung cancer, overall. However, stronger associations were seen among smokers, especially between UA and lung cancer in heavy smokers. A major strength of this study is its size, which enabled the capture of sufficient incident diagnoses, especially for lung cancer that has a low incidence rate, and gave sufficient statistical power to confirm an interaction between UA and smoking. Also, the authors showed in their analyses that reverse causation was an unlikely explanation for their findings. ${ }^{18}$ However, they acknowledge two limitations. The first, which is common to all primary care research databases using routinely collected data, is that information on potential confounders is often missing or incomplete. For example, in this study, it was not possible to control for socioeconomic status at the individual level. More importantly, given that UA concentrations are lower in smokers, detailed information on lifetime smoking history (pack years) was not available; hence, there is a real concern that residual confounding may, at least partly, explain the associations found. The second limitation is that the cohort of individuals selected for study was a subset of the total population, who had had serum UA measured for clinical reasons, such as symptoms suggestive of gout; hence the findings may not be generalisable to the wider population. Also, a key assumption in this study is that serum UA concentration is a reliable biomarker of UA concentration in the ELF; it would be interesting to know how strongly the two are correlated.

So where next with UA and respiratory disease? While attempts to replicate these latest findings in other prospective cohorts with more detailed information on confounders would be of interest, a potentially more informative and rigorous approach would be to use Mendelian randomisation (MR); this can strengthen causal inference by eliminating bias, confounding and reverse causation, which can thwart the interpretation of observational studies. ${ }^{19}$ Serum UA status is strongly influenced by genes, and a 
meta-analysis of genome-wide association studies has identified a number of common gene variants that predict serum UA concentrations. ${ }^{20}$ These variants can therefore be used as lifetime proxy measures of UA to determine whether UA is likely to be causally related to respiratory outcomes, although it is now appreciated that studies need to be very large in order to use the MR approach effectively. MR was recently used to investigate the relation between another endogenous antioxidant, bilirubin and lung function, and provided supportive evidence for a positive causal association. ${ }^{21}$ By contrast, the MR approach has been used to show that associations between UA and ischaemic heart disease and blood pressure found in observational studies are unlikely to be causal, and may be confounded by Body Mass Index which is likely to be causally related to UA. ${ }^{22}$ Horsfall and colleagues ${ }^{18}$ make the interesting suggestion that, even if serum UA is not causally related to respiratory disease, it might be capturing (through confounding) causal effects of risk factors such as adiposity and, therefore, could still be a useful biomarker in clinical practice to identify smokers who are at most risk of developing disease.

Ultimately, the only way to definitively establish whether higher UA concentrations are causally linked to a lower risk of respiratory disease is to carry out a randomised clinical trial. Horsfall et $a l^{18}$ suggest that trials involving supplementation with UA itself might be the way forward. The problem, of course, is that a high UA status may be a double-edged sword; even if raising serum UA concentrations were to achieve some benefit with respect to respiratory diseases such as COPD and lung cancer, it is also likely to increase the risk of gout. Dietary strategies to increase UA status, such as increasing consumption of purine-rich foods or fructose-rich fruits, run the same risk. ${ }^{23} 24$ Interestingly, observational studies have suggested that a high consumption of apples might protect against asthma, ${ }^{25} \mathrm{COPD}^{26}$ and lung cancer $^{27}$; if causal, this is likely to reflect the high fructose content of apples which increases plasma UA concentrations and antioxidant capacity, and not the high flavonoid content, as was previously assumed. ${ }^{28}$ Given that the prevalence of gout has been rising in the UK and the USA in recent decades, ${ }^{1}$ one might question the wisdom of interventions to increase UA status. On the other hand, by adopting a stratified prevention approach, it might be possible to avert an increase in gout, through initial screening and intervening to increase UA status only in individuals with low serum concentrations at baseline. I would argue, though, that we might do better to focus our efforts on trials to assess whether food-based interventions, aimed at increasing dietary antioxidant status, could safely improve the lung health of smokers, especially those in whom attempts at smoking cessation have failed.

\section{Competing interests None.}

Provenance and peer review Commissioned; internally peer reviewed.

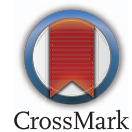

To cite Shaheen SO. Thorax 2014;69:978-979.

Published Online First 13 August 2014

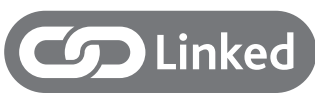

- http://dx.doi.org/10.1136/thoraxjnl-2014-205271

Thorax 2014;69:978-979.

doi:10.1136/thoraxjnl-2014-205751

\section{REFERENCES}

1 Richette P, Bardin T. Gout. Lancet 2010;375:318-28.

2 So A, Thorens B. Uric acid transport and disease. J Clin Invest 2010;120:1791-9.

3 Ames BN, Cathcart R, Schwiers E, et al. Uric acid provides an antioxidant defense in humans against oxidant- and radical-caused aging and cancer: a hypothesis. PNAS 1981;78:6858-62.

4 Kelly FJ, Blomberg A, Frew A, et al. Antioxidant kinetics in lung lavage fluid following exposure of humans to nitrogen dioxide. Am I Respir Crit Care Med 1996;154:1700-5.

5 Ciencewicki J, Trivedi S, Kleeberger SR. Oxidants and the pathogenesis of lung diseases. J Allergy Clin Immunol 2008;122:456-68.

6 Filaire E, Dupuis C, Galvaing G, et al. Lung cancer: what are the links with oxidative stress, physical activity and nutrition. Lung Cancer 2013;82:383-9.

7 Allen S, Britton JR, Leonardi-Bee JA. Association between antioxidant vitamins and asthma outcome measures: systematic review and meta-analysis. Thorax 2009:64:610-19.

8 Fogarty A, Lewis SA, Scrivener SL, et al. Oral magnesium and vitamin $C$ supplements in asthma: a parallel group randomized placebo-controlled trial. Clin Exp Allergy 2003;33:1355-9.

9 Holick CN, Michaud DS, Stolzenberg-Solomon R, et al. Dietary carotenoids, serum beta-carotene, and retinol and risk of lung cancer in the alpha-tocopherol, beta-carotene cohort study. Am J Epidemiol 2002;156:536-47.

10 The Alpha-Tocopherol, Beta Carotene Cancer Prevention Study Group. The effect of vitamin E and beta carotene on the incidence of lung cancer and other cancers in male smokers. $N$ Engl I Med 1994:330:1029-35.

11 Lawlor DA, Davey Smith G, Kundu D, et al. Those confounded vitamins: what can we learn from the differences between observational versus randomised trial evidence? Lancet 2004;363:1724-7.

12 Zmijewski JW, Lorne E, Zhao X, et al. Antiinflammatory effects of hydrogen peroxide in neutrophil activation and acute lung injury. $A m J$ Respir Crit Care Med 2009;179:694-704.

13 Sayin VI, Ibrahim MX, Larsson E, et al. Antioxidants accelerate lung cancer progression in mice. Sci Trans/ Med 2014:6:221ra15.

14 Bjelakovic G, Nikolova D, Gluud LL, et al. Mortality in randomized trials of antioxidant supplements for primary and secondary prevention: systematic review and meta-analysis. JAMA 2007;297: $842-57$

15 Schulz TJ, Zarse K, Voigt A, et al. Glucose restriction extends Caenorhabditis elegans life span by inducing mitochondrial respiration and increasing oxidative stress. Cell Metab 2007:6:280-93.

16 Shaheen SO, Jameson KA, Syddall HE, et al. The relationship of dietary patterns with adult lung function and COPD. Eur Respir J 2010;36: 277-84.

17 Wood LG, Garg ML, Smart JM, et al. Manipulating antioxidant intake in asthma: a randomized controlled trial. Am J Clin Nutr 2012;96:534-43.

18 Horsfall L, Irwin N, Petersen I. Serum uric acid and the risk of respiratory disease: a population-based cohort study. Thorax 2014;69:1021-6.

19 Davey Smith G, Ebrahim S. What can mendelian randomisation tell us about modifiable behavioural and environmental exposures? BM 2005;330:1076-9.

20 Kolz M, Johnson T, Sanna S, et al. Meta-analysis of 28,141 individuals identifies common variants within five new loci that influence uric acid concentrations. PLoS Genet 2009:5:e1000504.

21 Curjuric I, Imboden M, Adam M, et al. Serum bilirubin is associated with lung function in a Swiss general population sample. Eur Respir $J$ 2014:43:1278-88.

22 Palmer TM, Nordestgaard BG, Benn M, et al. Association of plasma uric acid with ischaemic heart disease and blood pressure: mendelian randomisation analysis of two large cohorts. BMJ 2013:347:f4262.

23 Choi HK, Atkinson K, Karlson EW, et al. Purine-rich foods, dairy and protein intake, and the risk of gout in men. N Engl I Med 2004;350:1093-103.

24 Choi HKC, Curhan G. Soft drinks, fructose consumption, and the risk of gout in men: prospective cohort study. BMJ 2008:336:309-12.

25 Shaheen SO, Sterne JA, Thompson RL, et al. Dietary antioxidants and asthma in adults: population-based case-control study. Am J Respir Crit Care Med 2001;164:1823-8

26 Miedema I, Feskens EJ, Heederik D, et al. Dietary determinants of long-term incidence of chronic nonspecific lung diseases. The Zutphen Study. Am J Epidemiol 1993;138:37-45.

27 Knekt $P$, Jarvinen $R$, Seppanen $R$, et al. Dietary flavonoids and the risk of lung cancer and other malignant neoplasms. Am J Epidemiol 1997; 146:223-30.

28 Lotito SB, Frei B. The increase in human plasma antioxidant capacity after apple consumption is due to the metabolic effect of fructose on urate, not apple-derived antioxidant flavonoids. Free Rad Biol Med 2004;37:251-8. 